\title{
The worth emphasizing surgical technique: ureteropyelostomy to manage urinary tract complications
}

\author{
Song-Yi Kim${ }^{1}$, Moonsang Ahn², Chanjoong $\mathrm{Choi}^{2}$ \\ ${ }^{1}$ Division of Transplantation, Department of Surgery, Chungnam National University Hospital, Daejeon, Korea \\ ${ }^{2}$ Department of Surgery, Chungnam National University School of Medicine, Daejeon, Korea
}

Background: Injury of the ureteral supplying artery usually causes ischemic injury of allograft ureter after renal transplantation. In the process of resolving the ischemic injury of the allograft ureter, because it can lead to allograft kidney damage, prompt treatment is necessary. The ureteropyelostomy using the recipient's ipsilateral native ureter is the best choice of several treatments that can salvage an allograft kidney. The aim of this report proves that the ureteropyelostomy using a native ureter is the surgical technique to be feasible management of urinary tract complications after renal transplantation.

Methods: A 41-year-old male undergone second renal transplantation in 2017. Initial transplantation was performed in 1992, with allograft in the right iliac fossa. Although no definite ischemic injury of allograft ureter was observed, injury of the ureteral supplying artery was observed during bench-work. The allograft ureter was anastomosed to the bladder and a double- $J$ catheter was inserted in the allograft ureter. After anastomosis of the allograft ureter, it was judged that blood supply was somewhat adequate. However, 18 days postoperative, he presented an abdominal pain with oozing on the surgical incision site. On computed tomography, there was a ureteral rupture and perirenal hematoma of the allograft. The ureteropyelostomy using a native ipsilateral ureter was performed successfully without any complications such as ureteral necrosis, urinary leakage, and urinary stricture. Results: After 3 years of follow-up, he had a well-functioning allograft with a serum creatinine level of $1.59 \mathrm{mg} / \mathrm{dL}$.

Conclusions: The ureteropyelostomy using a native ipsilateral ureter can be a safe and feasible surgical technique that treated urinary ischemic complications due to injury of the ureteral supplying artery and resulted in good graft and patient survival.

Corresponding author: Song-Yi Kim

E-mail: ray9060@gmail.com

(c) The Korean Society for Transplantation

This is an Open Access article distributed under the terms of the Creative Commons Attribution Non-Commercial License (http://creativecommons.org/licenses/by-nc/4.0/) which permits unrestricted non-commercial use, distribution, and reproduction in any medium, provided the original work is properly cited. 